\title{
Analogical scaffolding and the learning of abstract ideas in physics: Empirical studies
}

\author{
Noah S. Podolefsky and Noah D. Finkelstein \\ Department of Physics, University of Colorado at Boulder, Boulder, Colorado 80309, USA
}

(Received 12 March 2007; published 14 September 2007)

\begin{abstract}
Previously, we proposed a model of student reasoning which combines the roles of representation, analogy, and layering of meaning — analogical scaffolding [Podolefsky and Finkelstein, Phys. Rev. ST Phys. Educ. Res. 3, 010109 (2007)]. The present empirical studies build on this model to examine its utility and demonstrate the vital intertwining of representation, analogy, and conceptual learning in physics. In two studies of student reasoning using analogy, we show that representations couple to students' existing prior knowledge and also lead to the dynamic formation of new knowledge. Students presented with abstract, concrete, or blended (both abstract and concrete) representations produced markedly different response patterns. In the first study, using analogies to scaffold understanding of electromagnetic (EM) waves, students in the blend group were more likely to reason productively about EM waves than students in the abstract group by as much as a factor of 3 ( $73 \%$ vs $24 \%$ correct, $p=0.002$ ). In the second study, examining representation use within one domain (sound waves), the blend group was more likely to reason productively about sound waves than the abstract group by as much as a factor of $2(48 \%$ vs $23 \%$ correct, $p=0.002)$. Using the analogical scaffolding model we examine when and why students succeed and fail to use analogies and interpret representations appropriately.
\end{abstract}

DOI: 10.1103/PhysRevSTPER.3.020104

PACS number(s): 01.40.Fk

\section{INTRODUCTION}

This paper examines the interplay between two essential components of scientific reasoning, use of representation and analogy. Scientists use multiple representations (including verbal, graphical, and gestural) and easily shift among these representations. ${ }^{1,2}$ Scientists also frequently generate and use analogies to reason and communicate in day-to-day activities. ${ }^{3}$ Representation and analogy are often considered convenient ways of communicating concepts, but with the implication that concepts transcend these forms of discourse. This view is controversial. ${ }^{4}$ Previously, we have proposed a model of student reasoning which combines the roles of representation, analogy, and layering of meaning-analogical scaffolding. ${ }^{5}$ The present empirical studies build on this model to examine its utility. In this paper we present a series of results demonstrating the vital intertwining of representation, analogy, and conceptual learning in physics.

Analogy plays an essential role in scientific reasoning. ${ }^{6}$ Historical examples include Rutherford's planetary model of the atom ${ }^{7}$ or Maxwell's application of fluid theory to electromagnetism. ${ }^{8}$ Analogies are commonly used to teach students physics, as evidenced by the range of analogies used in physics textbooks. ${ }^{9}$ Research investigating students' use of analogy in physics gained momentum during the early 1980s. Early models proposed that an analogy can be treated as a mathematical mapping from a familiar conceptual structure, the base, to an unfamiliar conceptual structure, the target. ${ }^{10,11}$ Using this framing of analogy, Gentner and Gentner $^{12}$ found that students' reasoning about electric circuits was measurably influenced by the analogies that these students generated (i.e., flowing water vs moving object analogies), demonstrating that analogies constitute more than mere surface terminology; indeed, analogies generate inferences. However, this study also found that analogies that were taught to students were not as influential on these students' reasoning compared to analogies that the students generated themselves. Some question the notion of mapping between well defined structures, ${ }^{13,14}$ suggesting that this framing of analogy is insufficient to explain the complex (and often fragmented ${ }^{15,16}$ ) ways students reason. Nonetheless, researchers have confirmed that analogies can be productive for student learning, documenting cases where analogies generated by students ${ }^{17}$ and expert physicists ${ }^{18}$ can contribute to productive reasoning about physics problems. The idea that analogies can play a significant role in student reasoning is now well supported. Spiro et al. ${ }^{19}$ suggest teaching with multiple analogies in order to circumvent the drawbacks of single analogies (e.g., single analogies may be misleading or incomplete), especially when teaching complex and difficult topics. However, finding consistently productive ways of teaching with analogies remains a challenge to researchers. Teaching with analogies has met with mixed success $^{12,14}$ despite efforts to directly teach step-by-step processes, ${ }^{20,21}$ or to foster student use of analogies. ${ }^{22,23}$

Recently, however, some progress has been made toward identifying possible mechanisms of student analogy use. Several lines of research have suggested a tradeoff between within-domain and across-domain learning of abstract principles (e.g., modulo-3 arithmetic ${ }^{24}$ ). This tradeoff appears to be coupled to the concreteness of the representations used to teach students. ${ }^{24,25}$ Here, the concreteness of a representation is gauged by the degree to which the representation contains salient, information-rich features (e.g., a picture of a soccer ball is considered more concrete than a black dot meant to represent a generic rolling object). While researchers find that concrete representations are more productive for students learning within a single domain, the use of abstract representations better facilitates students productively using those ideas in a second domain. Along these lines, Van Heuvelen and $\mathrm{Zou}^{26}$ successfully used concrete representations to scaffold students interpretations of abstract (i.e., mathematical) representations when solving work-energy problems. Interestingly, Goldstone and Sakamoto ${ }^{25}$ report this tradeoff in learning for "low-achieving" students, but they find little or no such effect for "high-achieving" students. Sloutsky et al. ${ }^{24}$ find that irrelevant concreteness (e.g., pictures of insects used to represent mathematical entities) can 
hinder across-domain learning of mathematical principles. Surprisingly, Goldstone and Sakamoto ${ }^{25}$ find that even relevant concreteness can hinder across-domain learning.

These recent results parallel our own findings that representations can play a key role in teaching students with analogies. ${ }^{27}$ Based on these findings, we proposed a model of analogy use, analogical scaffolding, ${ }^{5}$ which describes mechanisms by which multiple analogies may be layered in order to learn abstract ideas. According to this model, concrete and abstract representations play key, complementary roles in this layering process. Using this model, we have modified curricular materials aimed to teach college physics students about electromagnetic (EM) waves by using analogies. Ambrose $e t$ al. ${ }^{28}$ have identified a number of student conceptual difficulties with EM waves in order to develop curricular interventions. These prior findings call for further study of how students can learn this challenging topic, particularly with regard to the use of wave representations.

In preliminary work ${ }^{5}$ we found that students taught EM waves concepts using materials based on analogical scaffolding outperformed students taught the same EM waves concepts without analogies on a pre-post assessment. In this paper, we describe two follow-up studies, the first examining student learning across multiple conceptual domains and the second examining student reasoning within a single domain. Primarily, we consider analogical scaffolding to be a cognitive model and these studies seek to examine the utility of this model to explain student reasoning and responses in educational environments.

In the first study across domains, we taught students about EM waves using analogies from multiple domains (wave-ona-string and sound waves). We explore the implications of varying the concreteness of the representations used to teach undergraduates in an algebra-based introductory physics course. In this study we ask, how does the model explain (and predict) student reasoning under different conditions, i.e., using different representational forms to teach? As a secondary goal, we investigate analogical scaffolding as a teaching intervention. To this end, we ask how closely the ideas of students taught with materials designed according to an analogical scaffolding framework align with canonical physics ideas. ${ }^{29}$

In the within-domain study, conducted in another algebrabased introductory physics course, we explore the implications for student reasoning and use of analogy by varying the concreteness of the representations used on a quiz within a single conceptual domain, sound waves. We pose the following research questions for this second study. (i) Previously, we found students associated different representations of a sound wave with various conceptions of sound waves (e.g., though sound is a longitudinal wave, students associate a sine wave representation of sound with transverse wave motion). ${ }^{27}$ In the present within-domain study we explore the directionality of this association and ask: do representations drive student use of analogy and, by proxy, conceptions of sound waves? (ii) How does varying the concreteness of representations affect students' reasoning about sound waves? The findings of this second study give insight into student learning of sound waves, one of three analogical scaffolds we pose as productive for layered student learning of EM waves in the preliminary ${ }^{5}$ and across-domain studies.

In both the across- and within-domain studies, we find students demonstrate markedly different response patterns to concept questions depending on the form of representations used to teach. In the across-domain study, the model makes accurate predictions about students' responses to EM waves concept questions and, furthermore, predicts which form of analogical scaffolding (of three investigated) is optimal for student learning of EM waves. In the within-domain study, we find representation can drive student reasoning about analogies. Furthermore, the analogical scaffolding model predicts how representational forms (and combinations of these) will affect students' ability to make productive use of an abstract representation of a sound wave, such as a sine wave.

\section{ANALOGICAL SCAFFOLDING}

We briefly outline analogical scaffolding theory-a more detailed account is presented in a prior paper. ${ }^{5}$ The analogical scaffolding model draws on theories of representation, ${ }^{30}$ conceptual blending, ${ }^{31}$ and layering of ideas. ${ }^{32}$ We draw on the work of Roth and Bowen ${ }^{30}$ to describe the relationship between a signifier, sign, the thing the sign refers to, referent, and a knowledge structure mediating the sign-referent relationship, schema. The word sign refers to external representations, such as text, graphs, equations, pictures, gestures, or utterances. Schemata (plural of schema) can be considered knowledge structures employed to interpret sign-referent relationships. Each system of sign-schema-referent can be considered a mental space, defined by Fauconnier and Turner as "a small conceptual packet constructed as we think and talk, for purposes of local understanding and action" (Ref. 31, p. 102). According to this model, productive schema elements are coupled to a sign whose surface-level features are associated with these schema elements [Along the lines of What-You-See-Is-What-You-Get, or WYSIWYG, as described by Elby (Ref. 33)]. For example, consider a picture of compressed and rarefied air particles, a sign representing the referent sound wave [Fig. 1(a)]. The sign and referent are coupled to a schema containing the elements "longitudinal" and "disturbance spreading through space" or "3D." Now, consider a sound wave represented by a sine wave [Fig. 1(b)]. The surface-level features of a sine wave are more tightly coupled to schema elements such as "transverse" and "2D" (a sine wave is generally drawn in a single plane). ${ }^{27} \mathrm{In}$ this case, the sine wave can cue a schema that is unproductive for sound. However, these two sign-referent-schema systems may be blended, whereby the sine wave comes to be coupled to a schema containing longitudinal and 3D. This process constitutes one layer within a conceptual domain. If, in a subsequent layer, the sine wave is coupled to the referent EM wave, the 3D longitudinal schema may be inherited by the EM wave mental space via another blend.

Blends combine mental spaces, linked by some connection between these spaces (e.g., the same sign or sometimes the same referent with different signs), and project selected schema elements (e.g., 3D) from these mental spaces to generate a blended space. Increasingly complex and abstract 

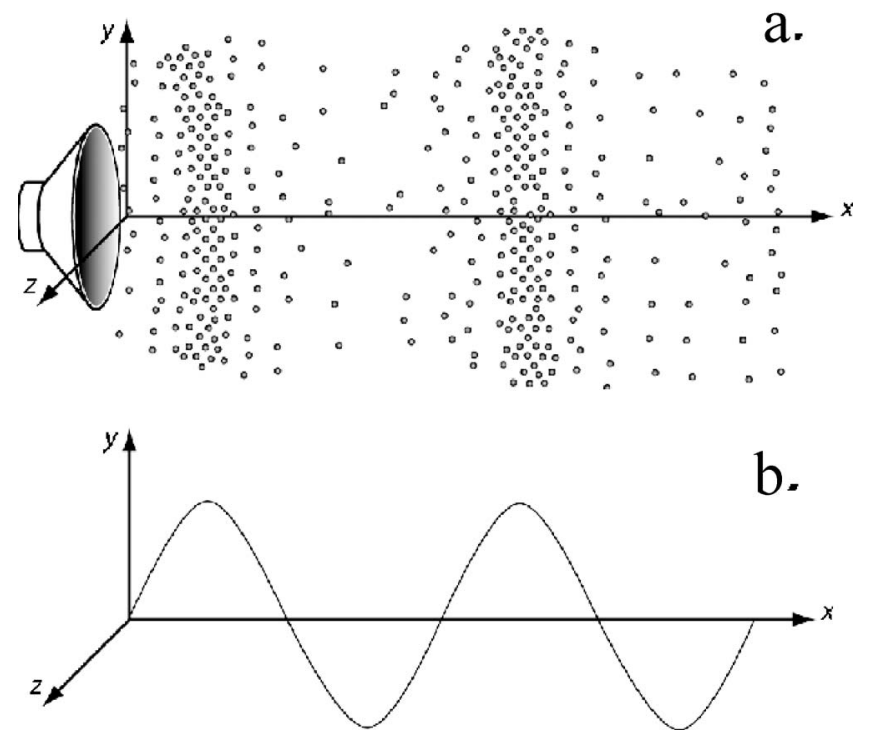

FIG. 1. A sound wave represented by a picture of compressed and rarefied air particles (a) and a sine wave (b).

ideas can be built up by a series of blended layers. ${ }^{32}$ For instance, a wave-on-a-string blends with sound waves, building up to EM waves. As a concrete example, in the next section we will describe a detailed application of this model to predict the outcomes of two empirical studies of student learning of $E / M$ waves by layered analogies of string and sound waves.

\section{STUDENT LEARNING ACROSS MULTIPLE DOMAINS}

\section{Methods}

\section{Classroom setup}

The participants in the across-domain study were $151 \mathrm{col}-$ lege students enrolled in the second semester of an algebrabased introductory physics course, focusing largely on electromagnetism. The first semester of this course included instruction on waves-on-a-string and sound waves as well as general wave properties. Prior to the tutorial activities described here, lectures and homework had covered electric and magnetic fields, but had not yet covered EM waves. This typical introductory course consisted of three 50-min lectures per week, used the Touger text, ${ }^{34}$ an online HW system, ${ }^{35}$ and included one 2-h recitation each week. Recitations generally included laboratory activities, but on occasion students worked on pencil and paper tutorials in lieu of hands-on experiments. Students generally worked in groups of 3-5. During these tutorial activities, the teaching assistant roamed the classroom answering students' questions and probing students' understanding of the materials to be learned. In the across-domain study, groups of students within a given recitation section were assigned to one of three treatment groups, denoted abstract, concrete, and blend. Table I lists the number of students $(N)$ for each group, summed over all recitation sections, and the average course grade for students in each group. We found no statistically significant difference
TABLE I. Across-domain study experimental groups.

\begin{tabular}{lcc}
\hline \hline Group & $N$ & Average course grade \\
\hline Abstract & 49 & $77.0 \%$ \\
Concrete & 51 & $76.5 \%$ \\
Blend & 51 & $78.6 \%$ \\
\hline \hline
\end{tabular}

between the average grades for the three groups $(p>0.3$, two-tailed $z$ test $\left.^{36}\right)$.

\section{Assessment before and after tutorials}

In recitation, students were issued pre- and post-tests on EM waves immediately before and after tutorials. These assessments were identical in all three treatment groups. The pre-test was administered at the beginning of recitation. These were collected, and students were then divided into three groups, each receiving a different version of a tutorial on EM waves, described below. After completing the tutorial, students were issued a post-test, identical to the pre-test. The only difference in treatment between the three groups was the type of representation used in the tutorial.

The assessments consisted of two open response questions, shown in Fig. 2. These questions are based on the materials used by Ambrose et al. ${ }^{28}$ to evaluate the Tutorial ${ }^{37}$ on EM waves, but were modified based on student interviews. ${ }^{38}$ Since these questions were open response, there was a large range of possible answers, and the likelihood of students guessing the correct answer was extremely low. Students were asked to explain their reasoning on each question. Note that these two questions require that students interpret the pictures in Fig. 2 as representing a snapshot in time of EM plane wave traveling to the right. Thus the correct answer to question 1 is $\mathrm{I}=\mathrm{J}=\mathrm{K}=\mathrm{L}$, since for a plane wave the magnitude of the electric field depends only on the $x$ coordinate. The correct answer to question 2 is $\mathrm{P}=\mathrm{Q}=\mathrm{R}=\mathrm{S}$, since, for a plane wave, the magnitude of the electric field depends on $x$, but since this is also a traveling wave, the time average signal is independent of $x$. Note that if question 2 had asked for the magnitude of the electric field at this instant in time, the correct answer would be $\mathrm{P}=\mathrm{Q}=\mathrm{R}>\mathrm{S}$, with $\mathrm{S}$ having magnitude zero. ${ }^{39}$

\section{Tutorials}

The tutorials used in the across-domain study were based in part on The Tutorials in Introductory Physics, ${ }^{37}$ but were modified to teach about EM waves using wave-on-a-string and sound wave analogies. ${ }^{40}$ The tutorials consisted of three main parts. Part 1 used a wave on a string to introduce transverse and traveling wave ideas. Part 2 used sound waves to introduce three-dimensional (3D) waves (close approximations to plane waves). Part 3 covered properties of EM plane waves, including basic wave properties such as frequency, wavelength, and amplitude, the interpretation of EM wave diagrams, and ways of detecting EM waves with an antenna.

The tutorials in each experimental group were nearly identical in content and wording, but differed in the repre- 
Question 1 The figure on the right shows an electromagnetic plane wave at one instant in time. The wave travels in the $+x-$ direction. Four points in space are labeled I,J,K,L.

For the instant shown, rank the points I, J, K, and L according to the magnitude of the electric field at these points, from largest to smallest. If the electric field is zero at any of these points, state that explicitly. For example, if you think $\mathrm{K}$ is the largest, and the rest are the same, you should answer $\mathrm{K}>\mathrm{I}=\mathrm{J}=\mathrm{L}$.

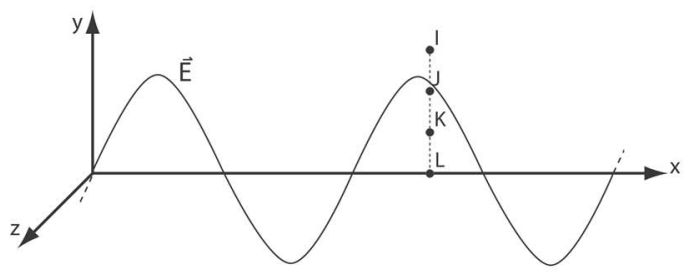

Explain your answer.

Question 2 The figure on the right shows an electromagnetic plane wave at one instant in time. The wave travels in the $+x-$ direction. Four antennas are labeled P,Q, R, and S. Antennas P, Q, and $\mathrm{S}$ lie in the $\mathrm{x}-\mathrm{y}$ plane. Antennas $\mathrm{P}, \mathrm{Q}$, and $\mathrm{R}$ have the same $\mathrm{x}-$ coordinate, but $\mathrm{R}$ is located out of the page in the $\mathrm{X}-\mathrm{Z}$ plane. All four antennas are oriented parallel to the $y$-axis.

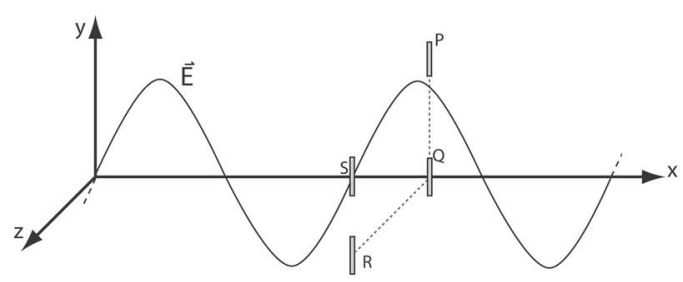

Rank the time-averaged signals received by the antennas $\mathrm{P}, \mathrm{Q}, \mathrm{R}$, and $\mathrm{S}$, from largest to smallest. If the time-averaged signal is zero at any of these points, state that explicitly. (Hint: the "time averaged signal" is the signal averaged over several cycles of the wave.)

Explain your answer.

FIG. 2. Questions given on the pre- and post-tests in the across-domain study.

sentations used. Figure 3 shows a subset of the representations of string, sound, and EM waves used in the abstract, concrete, and blend groups. The complete set of tutorials and surveys can be found in supplementary materials. ${ }^{40}$ The canonical wave representation is a sine wave, which we consider an abstract representation. ${ }^{5}$ In the abstract group, a sine wave is used consistently to represent string, sound, and EM waves. $^{41}$ The representations used in the concrete group include more salient features, for instance, showing compressed and rarefied air particles in a sound wave spread throughout space. In the blend group, students were presented with both abstract and concrete representations simultaneously. The tutorials included significant framing for students to make sense of these representations and learn about EM waves, generally in the form of Socratic questioning written into the tutorials. This framing (and wording) was nearly identical for the three treatment groups.

\section{Predictions}

We claim the analogical scaffolding model can make correct predictions of student answers (and associated reasoning) to explain the results of the across- and within-domain studies. Before outlining these predictions of analogical scaffolding, we explore alternative models.

According to one model, students' prior knowledge consists of relatively stable and well formed structures, akin to scientific theories, ${ }^{42-44}$ that are not strongly linked to particular contexts. ${ }^{45}$ When these ideas are non-canonical they are called misconceptions. ${ }^{33}$ This model predicts that many students will apply these theorylike ideas to conceptual ques- tions about EM waves, often resulting in students answering these questions incorrectly. The way misconceptions are changed is that students are presented with cases that conflict with their prior knowledge, and these students therefore reorganize their knowledge to align with this new case. This model has merit, and in fact the tutorials used in the acrossdomain study do address common student ideas about EM waves which may be inconsistent with experts' ideas. However, suppose students are presented with cases that conflict their prior knowledge in a tutorial, but under different representational conditions, as is the case in the present study. This model of student learning alone does not predict nor does it explain how students will reorganize their knowledge under these different conditions. In other words, this model is not sensitive to context-it predicts that students will reorganize their knowledge in all three treatment groups (abstract, concrete, and blend), but does not make specific predictions about how students will learn differently in the three groups. Determining which condition is optimal is purely an empirical endeavor. To be sure, curricular materials based on this model can be extremely productive in bringing students ideas closer to experts'. However, we seek mechanisms which are sensitive to context and can therefore predict and explain how using different representations and analogies impact student learning differently.

Elby ${ }^{33}$ proposes one such mechanism of interpreting representations, what-you-see-is-what-you-get or WYSIWYG. When WYSIWYG is activated, students interpret representations literally, for instance, they may interpret a graph shaped like a hill literally as a hill (even if this graph is of velocity vs time). We predict that on questions like those in Fig. 2, 


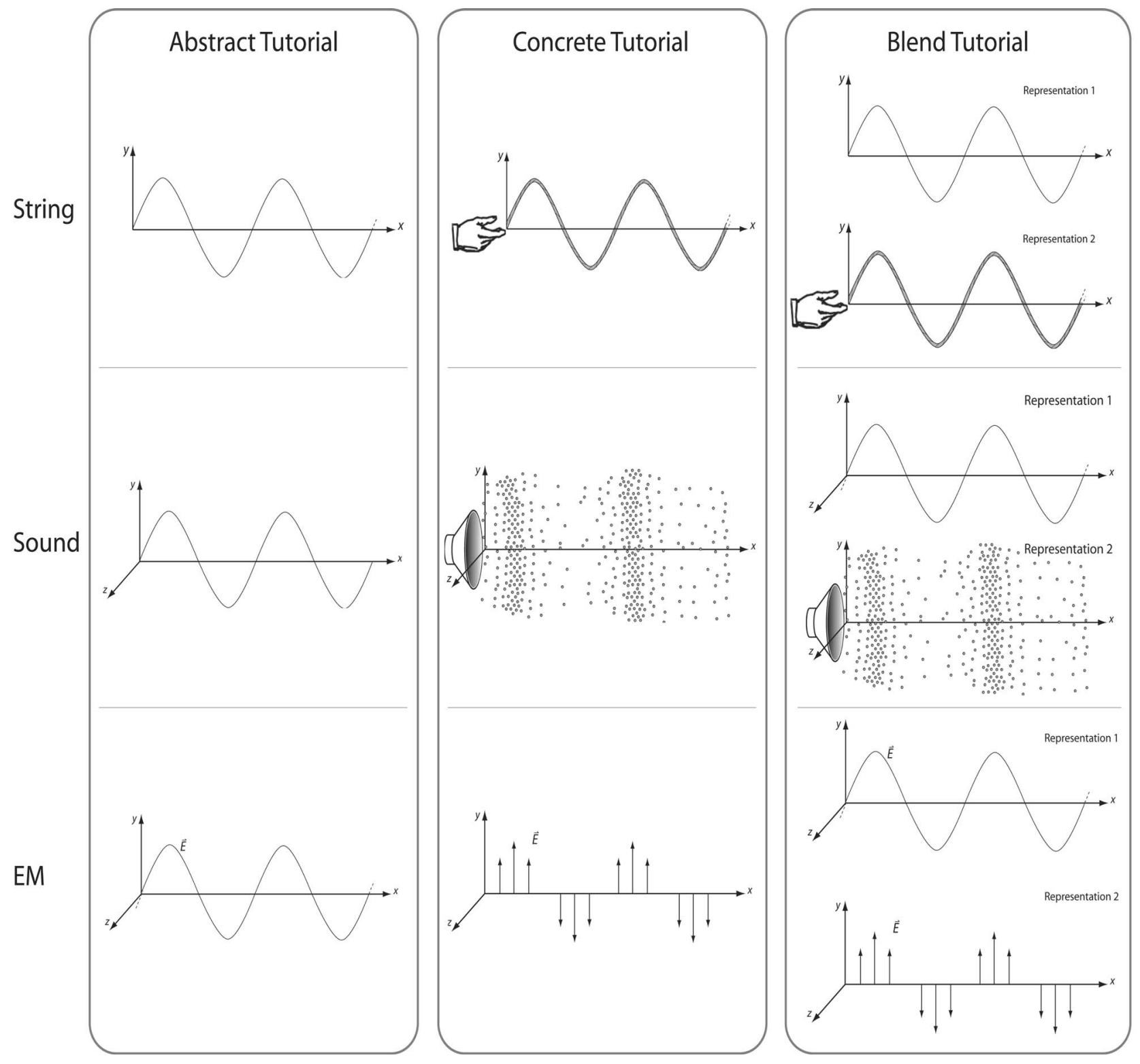

FIG. 3. Examples of representations of a wave on a string, sound wave, and EM wave used in the abstract, concrete, and blend tutorials in the across-domain study. The complete set of representations can be found in the supplementary materials (Ref. 40).

students who apply WYSIWYG will treat the sine wave literally as moving up and down in the $x-y$ plane. This prediction is based most significantly on the particular representations used on the pre- and post-tests. However, because WYSIWYG is so strongly tied to these representations, it may fail to predict when students will not use WYSIWYG. WYSIWYG alone would predict no differences between the three conditions on the pre-test, but also on the post-test, since all three groups had exactly the same questions with the same representations. One explanation for why students would not use WYSIWYG is simply that student answers have some randomness to them, or alternatively that students who answer with a non-WYSIWYG interpretation (but correctly) simply "get it." Analogical scaffolding uses WYSIWYG as one mechanism of reasoning from representations, but additional mechanisms are required to explain when and why students will not use WYSIWYG, especially when stu- dents use other interpretive strategies productively. In other words, analogical scaffolding explains why some students appear to get it, but also why students who do not answer concept questions correctly may nonetheless answer these questions in predictable ways.

Studies of analogy suggest that while potentially powerful, students often fail to use an analogy productively if at all. Therefore we might expect students to directly apply what they have learned about EM waves during the tutorials to the post-test question, but not use ideas from string and sound waves. That is, students do not apply the analogies provided. In this case, we might expect differences between the abstract, concrete, and blend groups based on the treatment of EM waves in the tutorials. However, WYSIWYG applied to EM wave representations (both sine wave and vectors) does not lead in any obvious way to developing $3 \mathrm{D}$ or traveling wave ideas about EM waves, since these ideas 

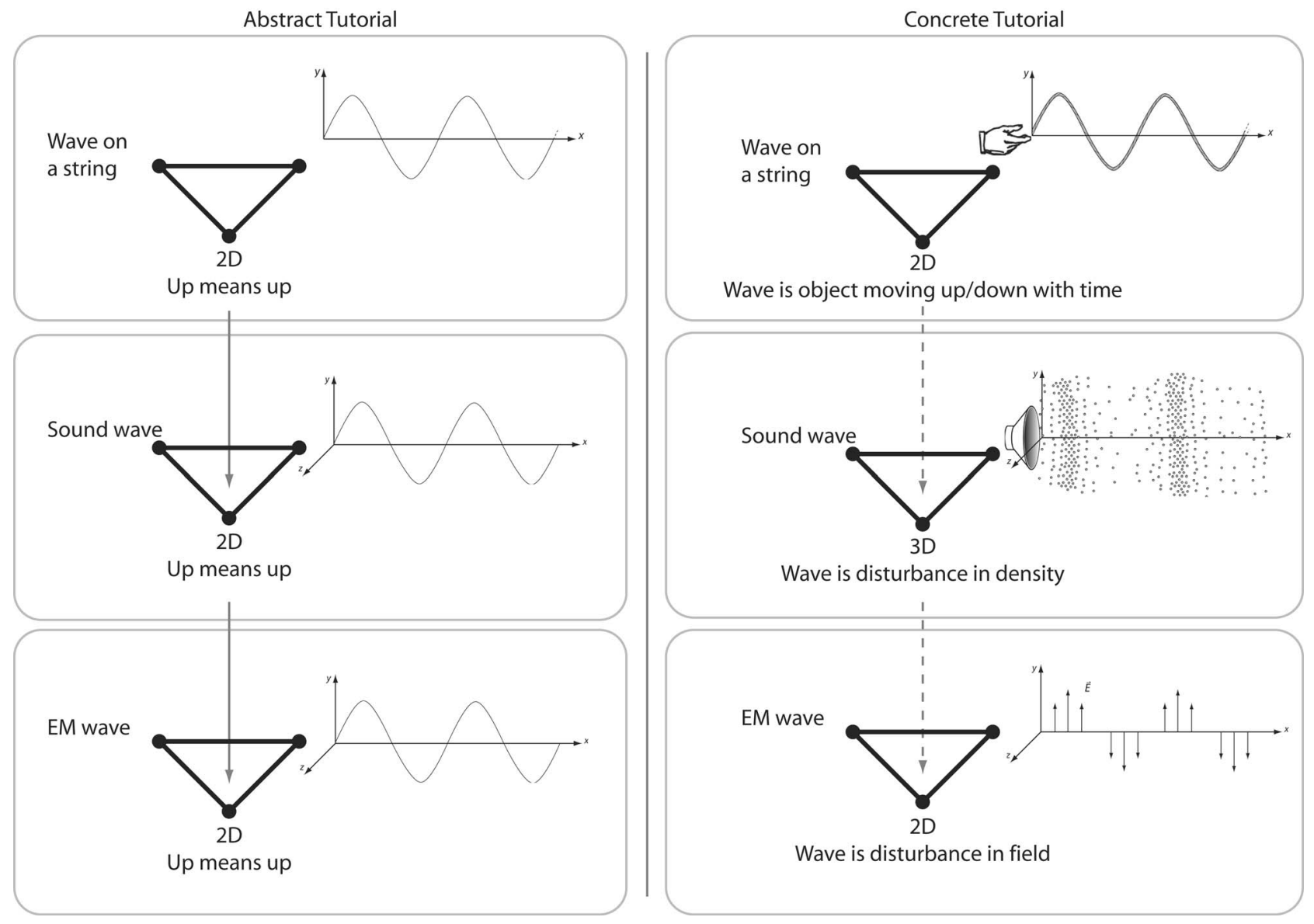

FIG. 4. Analogical scaffolding schematics for the abstract (left) and concrete (right) tutorials.

were taught only for sound and wave on a string. Again, if students only directly applied their (possibly reorganized) knowledge of EM waves to the post-test question, we would not expect differences between the three groups since these questions specifically test students knowledge of 3D and traveling waves.

These alternative models can be reformulated according to three hypotheses on the role of representations in student learning EM waves via analogical scaffolding:

(i) The null hypothesis. Student learning depends mostly on prior knowledge and reorganizing this knowledge to align with a new conflicting case. Representations and student learning are largely independent, both within and across domains, and we should therefore expect no differences between the three groups in both the across- and within-domain studies since the only variation between conditions was the representations used.

(ii) The weak hypothesis. Representations do couple to students' prior knowledge along the lines of WYSIWYG, but this coupling is only dependent on the immediate context. Observed differences between the treatment groups in the within-domain study would be sufficient to confirm this hypothesis, since students in this study received different representations on the assessment. However, this hypothesis would also predict no measurable differences between treatment groups in the across-domain study, since all students received the same representations on the assessments. (iii) The strong hypothesis. Representations not only cue existing prior knowledge, but also lead to the dynamic formation of new knowledge. This process is strongly dependent on the form and presentation of the representations. To confirm this, we would need to observe differential response patterns between the treatment groups on assessments in both the within- and the across-domain study. Differential response patterns would show that the representations used to teach had different effects on how students learn new interpretations of the representations in Fig. 2.

We now apply analogical scaffolding theory to predict the outcomes of the across-domain study. Three possible sequences of representational cueing, blending, and projection are shown in Figs. 4 and 5. The sign (representation) is shown at the upper right node of each triangle, referent at the upper left node, and schema at the bottom node. Figure 4 represents the abstract tutorial, with only sine wave representations used, and the concrete tutorial, with concrete representations used. In the abstract tutorial, the surface level interpretations of the sine wave would lead students to use a schema including the features $2 \mathrm{D}$ and "up means up." 27 This schema is projected through to EM waves, cued in each layer by the same sine wave. Alternatively, in the concrete tutorial, surface level interpretations lead students to apply different schemata to string, sound, and EM waves. However, students are predicted not to project these schematic elements (e.g., 3D) from one domain to the next as often as in the blend 


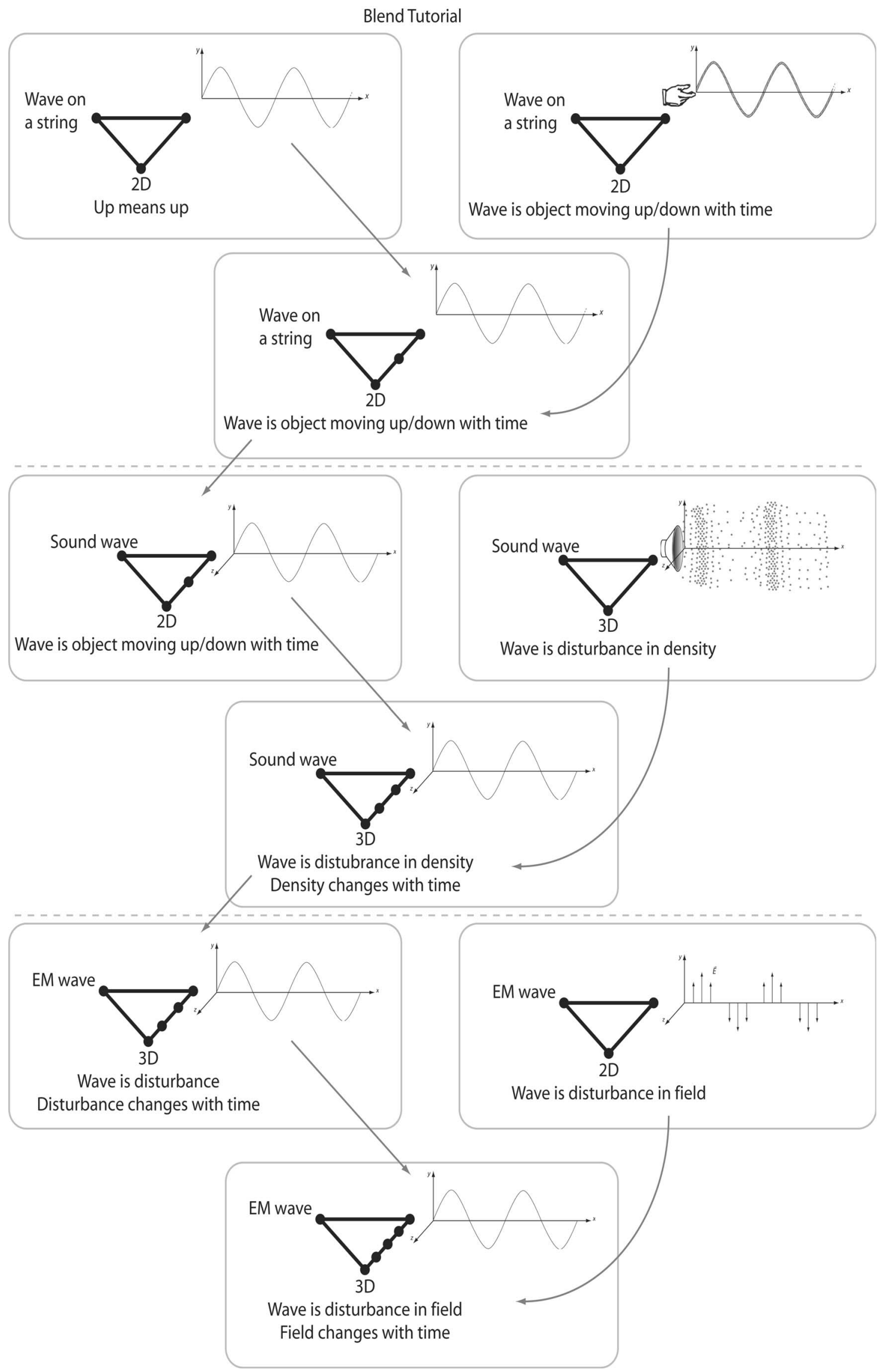

FIG. 5. Analogical scaffolding schematic for the blend tutorial. Dashed lines delineate string, sound, and EM wave domains. 
group since there is no corresponding sign (e.g., sine wave) to cue blended schemata in subsequent layers. This predicted lack of projection, one possible approach students may take, is represented by dashed arrows in Fig. 4.

Note that in Fig. 4, schemata are presented as separate, unblended pieces. In these cases, WYSIWYG operates within each piece, cueing schemata that are tightly coupled to signs. In the blend tutorial (Fig. 5), these schemata are cued by signs in a similar fashion to the abstract and concrete tutorials, but in this case schemata blend. Blended schemata then project through each layer and subsequently reblend. Each blend corresponds to an additional node between the sign (upper right) and schema (bottom) nodes of the resulting triangles. The final blend for EM waves has three nodes, corresponding to three prior blends. Note that in the blend treatment group, the schemata resulting from each blend are non-WYSIWYG.

This model predicts that students in the abstract group will be most likely of the groups to apply 2D, "up means up" objectlike schema elements when answering the post-test questions. This reasoning would be consistent with the answer I $>\mathrm{J}>\mathrm{K}>\mathrm{L}$ on question 1. (This reasoning would also be consistent with a number of incorrect answers to question 2 based on "up means up" reasoning, for instance $P>Q=S$ $>\mathrm{R}, \mathrm{P}>\mathrm{Q}>\mathrm{S}>\mathrm{R}, \mathrm{P}>\mathrm{Q}=\mathrm{R}=\mathrm{S}$, etc.) Students in the blend group will more likely apply $3 \mathrm{D}$, time-varying schema elements, and treat the sine wave as representing an abstract quantity (e.g., field) rather than treating the sine wave as an object (i.e., an object that goes up and down in space like a string). This reasoning would be consistent with $\mathrm{I}=\mathrm{J}=\mathrm{K}=\mathrm{L}$ on question 1 and $\mathrm{P}=\mathrm{Q}=\mathrm{R}=\mathrm{S}$ (both traveling and $3 \mathrm{D}$ ) or $\mathrm{P}=\mathrm{Q}=\mathrm{R}>\mathrm{S}$ (only $3 \mathrm{D}$ ) on question 2 . Students in the concrete group will fall somewhere in between, having been exposed to the essential schema elements, but not led to create blends of these schemata. Pre-test results for all groups would be most similar to the post-test predictions for the abstract group, since students are asked to answer questions about a sine wave representation of an EM wave before instruction. Note that we do not expect these coarse categorizations to describe individual students, as individual student resources and reasoning are sure to vary. We therefore note that these predictions are probabilistic and we predict trends in students reasoning for statistically robust numbers of students. (Our studies use $N>100$ subjects.)

\section{Results \\ Question 1}

Key results from the pre- and post-tests in the acrossdomain study are shown in Figs. 6 and 7. On pre-test question 1 (Fig. 6), less than $10 \%$ of students answered correctly $(\mathrm{I}=\mathrm{J}=\mathrm{K}=\mathrm{L})$, with no statistically significant difference between groups $(p>0.7)$. On the post-test, the scores for all groups increased, but, compared to the abstract group, students in the concrete and blend groups were more likely to produce the correct answer by factors of more than 2 and 3 , respectively $(p<0.01)$. As predicted, the concrete group lies in between, with the blend group marginally more likely to produce the correct answer (a difference of 16\%, $p<0.1$ ).

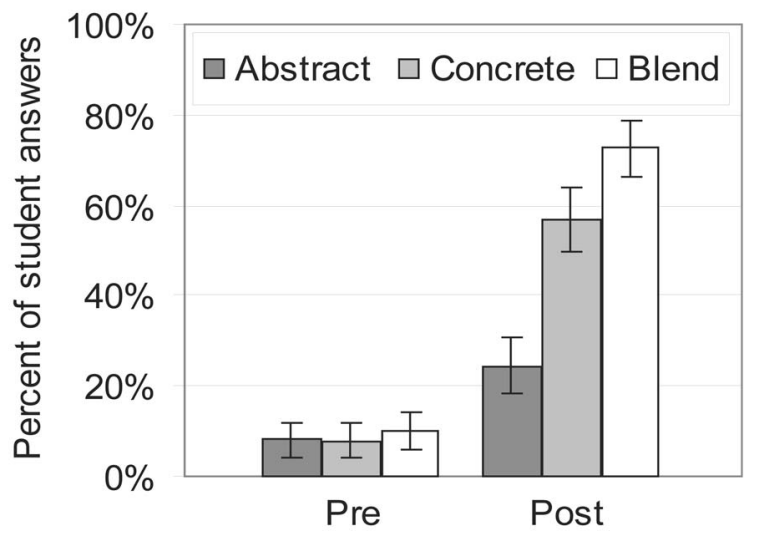

FIG. 6. Fraction of correct answers on pre-post question 1 from the across-domain study. Error bars represent \pm the standard error on the mean.

The most popular (incorrect) answer on pre-test question 1 was I $>\mathrm{J}>\mathrm{K}>\mathrm{L}$, answered by approximately $24 \%$ of students. This pre-test result was the same, statistically, in all three groups $(p>0.3)$. On the post-test, less than $10 \%$ of student in the concrete and blend groups answered $\mathrm{I}>\mathrm{J}$ $>\mathrm{K}>\mathrm{L}$, while $18 \%$ of students in the abstract group wrote this answer. This result is statistically significantly different between abstract and blend groups $(p=0.055)$, but not between abstract and concrete groups $(p=0.22)$. Another somewhat popular answer, $\mathrm{J}>\mathrm{I}=\mathrm{K}>\mathrm{L}$, was produced by $18 \%$ of students on the pre-test. On the post-test, $22 \%$ of students in the abstract group answered $\mathrm{J}>\mathrm{I}=\mathrm{K}>\mathrm{L}$, while less than $5 \%$ of students in the concrete and blend groups wrote this answer, significantly less than the abstract group $(p<0.01)$. We note that these incorrect answers are similar to those observed by Ambrose et al. ${ }^{28}$

We coded student explanations of reasoning on question 1 according to five categories, shown in Table II. The number of students $(N)$ producing each answer is shown above the corresponding answer, with the percentage of students producing that answer binned into each reasoning category below. We used an emergent coding scheme based on students' answers. Proximity to line corresponds to primitive reasoning ${ }^{46}$ such as "closer is more," i.e., interpreting the sine wave as an object and reasoning that a closer proximity to this object means stronger field. Read as graph corresponds to primitive reasoning such as "higher is higher," i.e., interpreting the positions of the points as heights on a graph of amplitude. ${ }^{47}$ Same $X$ position corresponds to reasoning that the magnitude of the E-field depends only on the $x$ coordinate. Sound words indicates usage of words related to sound, such as pressure and density. Other corresponds to explanations that were rare, unintelligible, or left blank. We group all students together on the pre-test, since these responses came before any differential instruction.

On the pre-test, and across all three groups on the posttest, we find similar patterns in Table II. The results are generally diagonalized, suggesting a strong association between answer and reasoning. ${ }^{48}$ Students answering correctly ( $\mathrm{I}=\mathrm{J}$ $=\mathrm{K}=\mathrm{L}$ ) used Same $X$ position reasoning, students answering $\mathrm{I}>\mathrm{J}>\mathrm{K}>\mathrm{L}$ used Read as graph reasoning, and students 
TABLE II. Coded student explanations on question 1 from the across-domain study pre- and post-tests.

\begin{tabular}{|c|c|c|c|c|c|c|c|c|c|c|c|c|}
\hline & \multicolumn{3}{|c|}{ All Groups Pre } & \multicolumn{3}{|c|}{ Abstract Post } & \multicolumn{3}{|c|}{ Concrete Post } & \multicolumn{3}{|c|}{ Blend Post } \\
\hline $\mathrm{N}=$ & 13 & 36 & 35 & 12 & 10 & 13 & 28 & 5 & 2 & 36 & 3 & 0 \\
\hline Answer: & $\begin{array}{l}\mathbb{\pi} \\
\stackrel{\pi}{\|} \\
\mathbb{\|}\end{array}$ & $\frac{\vec{\lambda}}{\stackrel{\Delta}{\Delta}}$ & $\begin{array}{l}\vec{\lambda} \\
\frac{\Delta}{\Delta} \\
\frac{\lambda}{\Delta}\end{array}$ & $\frac{\pi}{\pi}$ & $\begin{array}{l}\vec{\Delta} \\
\frac{\Delta}{\Delta}\end{array}$ & $\begin{array}{l}\vec{A} \\
\frac{\hat{A}}{\Delta}\end{array}$ & $\frac{\pi}{\frac{\pi}{\|}}$ & $\begin{array}{l}\vec{\lambda} \\
\stackrel{\Delta}{\Delta} \\
\stackrel{\Delta}{\Delta}\end{array}$ & $\frac{\vec{A}}{\stackrel{\Delta}{n}}$ & $\begin{array}{l}\pi \\
\underset{\pi}{\pi}\end{array}$ & $\begin{array}{l}\overrightarrow{\hat{A}} \\
\hat{\Delta} \\
\hat{\Delta}\end{array}$ & $\begin{array}{l}\vec{\lambda} \\
\stackrel{\Delta}{\|} \\
\stackrel{\Delta}{\Delta}\end{array}$ \\
\hline Proximity to Line & $0 \%$ & 0 & 100 & $0 \%$ & 0 & 100 & $0 \%$ & 0 & 100 & $0 \%$ & 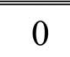 & \\
\hline Read as Graph & 0 & 81 & 0 & 0 & 60 & 0 & 0 & 80 & 0 & 0 & 100 & \\
\hline Same X Position & 54 & 0 & 0 & 67 & 0 & 0 & 50 & 0 & 0 & 69 & 0 & \\
\hline Sound Words & 0 & 0 & 0 & 33 & 10 & 0 & 36 & 0 & 0 & 47 & 0 & \\
\hline Other & 46 & 19 & 0 & 17 & 20 & 0 & 21 & 20 & 0 & 6 & 0 & \\
\hline
\end{tabular}

answering $\mathrm{J}>\mathrm{I}=\mathrm{K}>\mathrm{L}$ used Proximity to line reasoning. Zero students in the blend group answered $\mathrm{J}>\mathrm{I}=\mathrm{K}>\mathrm{L}$ on the post-test. Notably, the fraction of students using Sound words increased from zero on the pre-test to more than $33 \%$ on the post-test. Importantly, students who answered question 1 correctly used similar reasoning across all three groups. We note that very few students in the concrete and blend groups answered $\mathrm{I}>\mathrm{J}>\mathrm{K}>\mathrm{L}$ or $\mathrm{J}>\mathrm{I}=\mathrm{K}>\mathrm{L}$, but that for these few students, their reasoning patterns match those of students in the abstract group. However, combining these results with Fig. 6, we find that students in the concrete and blend groups were significantly more likely to answer correctly and thus more likely to use Same $X$ position reasoning on the post-test compared to students in the abstract group.

\section{Question 2}

Figure 7 shows the results for question 2 . On the pre-test, less than $10 \%$ of students answered correctly $(\mathrm{P}=\mathrm{Q}=\mathrm{R}=\mathrm{S})$, with no statistically significant difference between groups $(p>0.6)$. This question proved challenging for students, and less than $18 \%$ answered correctly on the post-test (no difference between groups, $p>0.3$ ). We did, however, find significant results on another popular answer, $\mathrm{P}=\mathrm{Q}=\mathrm{R}>\mathrm{S}$, which would be correct if the question had asked for the magnitude of the E field at the instant shown. We consider this answer partially correct, since it includes the plane wave feature of EM waves, but not the time average feature. On the pre-test, students in the abstract group produced the partially correct answer more often than the other two groups $(p<0.06)$. This trend reversed on the post-test-the blend group produced the partially correct answer significantly more often than both the concrete group and abstract group $(p<0.02)$. The concrete group was marginally more likely to produce the partially correct answer than the abstract group $(p=0.09)$. Interestingly, the fraction of students in the abstract group answering partially correct was unchanged from pre-to post-test, but the majority of these students answering partially correct on the post-test were not the same students that selected the partially correct answer on the pre-test. ${ }^{49}$ The only other answer produced by more than $10 \%$ of students on the post-test was $\mathrm{S}>\mathrm{P}>\mathrm{Q}>\mathrm{R}$. $14 \%$ of students in the abstract group produced this answer, while almost none of the concrete or blend group did (2\% and $0 \%$ respectively, significantly less than the abstract group, $p<0.05)$.

\section{Further analysis and follow-up studies}

As a direct measure of changes in student reasoning, we found that $40 \%$ of students in the abstract group did not change their answers to question 1 from pre to post, while less than $20 \%$ of students in the other two groups did not change their answers from pre to post. On question $2,15 \%$ of the abstract group did not change their answers from pre to post, while less than $8 \%$ of students in the other groups answered the same way from pre to post.

Two weeks after the EM waves tutorial, students were issued an online quiz with one question directly targeting the EM wave concepts in the tutorial. Lectures and homework during this two week interval included material covering EM waves. On this follow-up question, while the fractions of

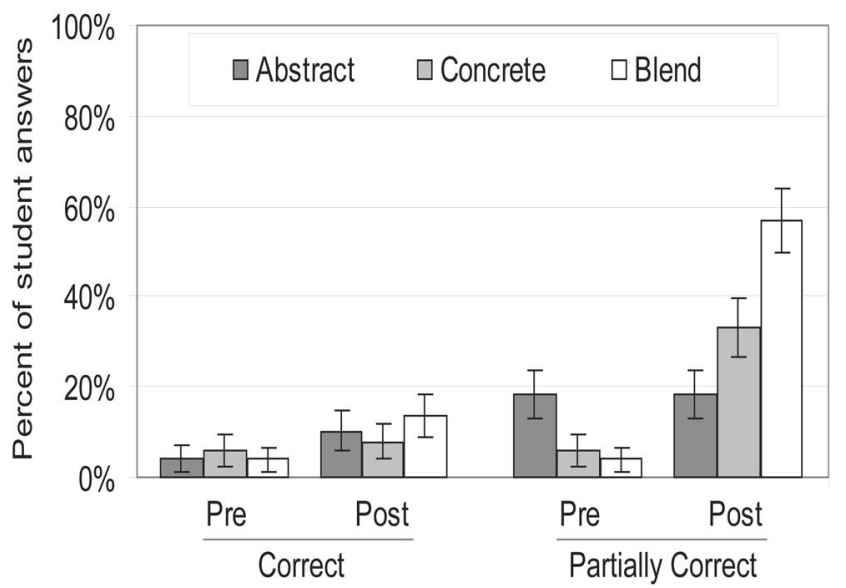

FIG. 7. Fraction of correct $(\mathrm{P}=\mathrm{Q}=\mathrm{R}=\mathrm{S})$ and partially correct $(\mathrm{P}=\mathrm{Q}=\mathrm{R}>\mathrm{S})$ answers on pre-post question 2 from the across domain study. 
correct responses from students in the blend and concrete groups were not significantly different $(p=0.2)$, both of these groups chose the correct answer significantly more often than students in the abstract group $(p<0.05)$. Evidently, the instruction during this two week gap, the same for all students, did not help students taught only with abstract representations catch up with students who were taught with concrete representations (or both abstract and concrete in the blend group).

Goldstone and Sakamoto ${ }^{25}$ found that varying the concreteness of representations affected the learning of lowperforming students, but that high-performing students were relatively unaffected by this variation. We explored the possibility of finding a similar result by analyzing the preceding results for the upper and lower halves of the class (high and low performers, respectively) based on overall course grade. Overall, we found no significant differences between the prepost results of high and low performers across all three treatment groups, with one exception. The response patterns of high performers in the concrete group were the same as students in the blend group $(p>0.1)$, while low-performers in the concrete group produced responses that were marginally different from the blend group $(p<0.1) .{ }^{50}$ Thus we find that teaching with multiple representations (blend group) may benefit low-performing students compared to teaching with single concrete representations. At the same time, teaching with single abstract representations appears to limit learning even for typically high-performing students in this population studied.

\section{Discussion of the across-domain study}

In the across-domain study, three versions of a tutorial on EM waves used varying representations according to a model of analogical scaffolding, and the model was employed to predict trends in student learning with these tutorials. We demonstrated applications of the model to construct schematic representations of these tutorials (Figs. 4 and 5) and to make specific predictions about student response patterns under these three conditions. The across-domain study demonstrated two key findings on student learning with analogies. (i) Across several conceptual questions on EM waves, students taught with abstract, concrete, or blend representations produced substantially different response patterns on these questions. (ii) We found that students' reasoning about and answers to these questions were associated in similar ways for the abstract, concrete, and blend groups. Thus while students produced consistent associations between reasoning and response patterns, the representations used to teach appear to drive different types of reasoning (and thus responses). These results bolster the utility of the analogical scaffolding model to predict differences in acrossdomain student learning under different conditions.

Analogical scaffolding may be employed to analyze cases where treatments led students to produce responses associated with noncanonical ideas about EM waves. Abstract representations may not provide students with the useful schemata for string and sound waves to apply to EM waves. Rather, we found students used surface-level reasoning to interpret the meanings of these representations (see Table II), leading these students to apply unproductive schemata to EM waves. For instance, a surface-level interpretation of a sine wave leads students to read string, sound, and EM wave diagrams as "higher means higher" or "closer means stronger." Concrete representations do provide productive string and sound wave schemata for students, and we observe students applying these schemata to EM waves sometimes. However, without an abstract representation to blend, these schemata are less likely to be applied to EM waves compared to when students are presented with both concrete and abstract (i.e., blend) representations together. Notably, the concrete group responses are closer to the blend group than the abstract, especially for the high-performing students. This is consistent with the hypothesis that high-performing students in the concrete group are using blends on their own.

\section{STUDENT REASONING WITHIN A SINGLE DOMAIN}

\section{Methods \\ Classroom setup}

The participants in the within-domain study were 353 college students enrolled in the first semester of an algebrabased introductory physics course, focusing on Newtonian mechanics. This is the same course sequence as the acrossdomain study and has a similar structure to the secondsemester course described above. Since both studies took place during the same semester, the two studies involved different students. Students were again assigned to one of three treatment groups, denoted as abstract, concrete, and blend groups. All students in a given recitation were assigned to the same group and issued a quiz on sound waves. In recitation the week prior to this study, students had completed a laboratory activity on sound. This activity involved using a microphone to take measurements of sound waves inside a long tube. ${ }^{51}$ Lectures prior to this study had covered mechanical waves, but students had received no explicit instruction on plane (3D) waves. Differences among teaching assistants (TA's) were mitigated by distributing the treatment group assignments evenly among the TA's. Table III lists the number of students $(N)$ for each group, compiled over all recitation sections, and the average course grade for students in each group. The average grade for the concrete group was not statistically different from the other two groups ( $p$ $>0.27)$. The blend group's grades were higher than the abstract group, with weak significance $(p=0.064)$. This weak difference does not account for the variance we find in our results, and all following significant results remain so when normalized to account for this small variation in student grade.

TABLE III. Within-domain study experimental groups.

\begin{tabular}{lcc}
\hline \hline Group & $N$ & Average course grade \\
\hline Abstract & 120 & $74.3 \%$ \\
Concrete & 114 & $76.1 \%$ \\
Blend & 119 & $78.7 \%$ \\
\hline \hline
\end{tabular}


Consider the following four analogies for a sound wave:

a. A crowd in a stadium doing "the wave".

b. A wave on a string.

c. A long row of people passing footballs from person to person.

d. A wave made with a stretched slinky.

e. Something else.

Which analogy or analogies (you may use more than one) seem the best for describing a sound wave? Explain your reasoning.

Note there is no "correct answer" - it is up to your interpretation.

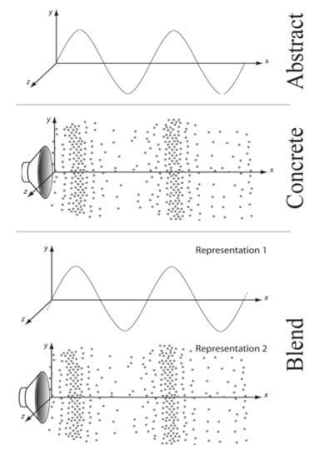

FIG. 8. Question 1 from the sound waves quiz. Each group (abstract, concrete, and blend) received a different representation shown on the right.

\section{Sound waves quiz}

Students were issued a quiz on sound waves at the beginning of recitation. ${ }^{40}$ The quizzes for the abstract, concrete, and blend groups were nearly identical in content and wording, but differed in the representations used. The quiz contained three multiple-choice questions. Question 1 presented an abstract, concrete, or blend representation of a sound wave, corresponding to each treatment group, directly to the right of the question statement as shown in Fig. 8. The text of question 1 was the same for all treatment groups. The analogy choices in question 1 draw on students' conceptions of a sound wave described by Hrepic. ${ }^{52}$ Note the representations in Fig. 8 are the same as those shown in the middle of Fig. 3 for the sound part of the tutorial in the across-domain study.

Questions 2 and 3 are shown in Figs. 9 and 10, respectively. For the abstract and blend groups, the representations used on question 2 were the same (a sine wave). For the concrete group, question 2 used a picture showing air particles. The wording of question 2 was the same for all three treatment groups. Question 3 was identical for all three groups in both wording and representation used. Questions 1 and 2 both appeared on the first page of the quiz, and question 3 appeared on a separate second page.

\section{Predictions}

Following our discussion of alternate models in the across-domain study, we briefly outline what these alternate models might predict for the within-domain study. The analysis is similar. Note that the only differential conditions for students occurred during the assessment. If we assume students' ideas are relatively stable and theorylike, a misconceptions model would predict no differences between the groups. WYSIWYG would predict differences between the groups on question 1 -students in the abstract group will differentially choose transverse wave analogies, while students in the concrete group will more likely choose longitudinal wave analogies. However, in the blend group, students are presented with two representations, and WYSIWYG does not provide a mechanism for why students would apply WYSIWYG to one representation over another. Therefore, using WYSIWYG alone, we would predict an even distribution of transverse and longitudinal wave analogies in the

The diagram on the right shows four points (labeled 1-4) in space in front of a speaker. The points are separated by a small distance (less than the size of the speaker.) Points 1,2 , and 4 lie in the $x$-plane. Point 3 has the same $x-$ coordinate as 1 and 2, but lies out of the page (in the zdirection).

Which of the following is the best ranking of magnitude of the pressure at the four points? Note the pressure is proportional to the density of the air particles.
a. $1>2=4>3$
b. $1=2=3>4$
c. $4>1=2=3$
d. $1=2=4>3$
e. $1=2>4>3$
f. $1=2>4=3$
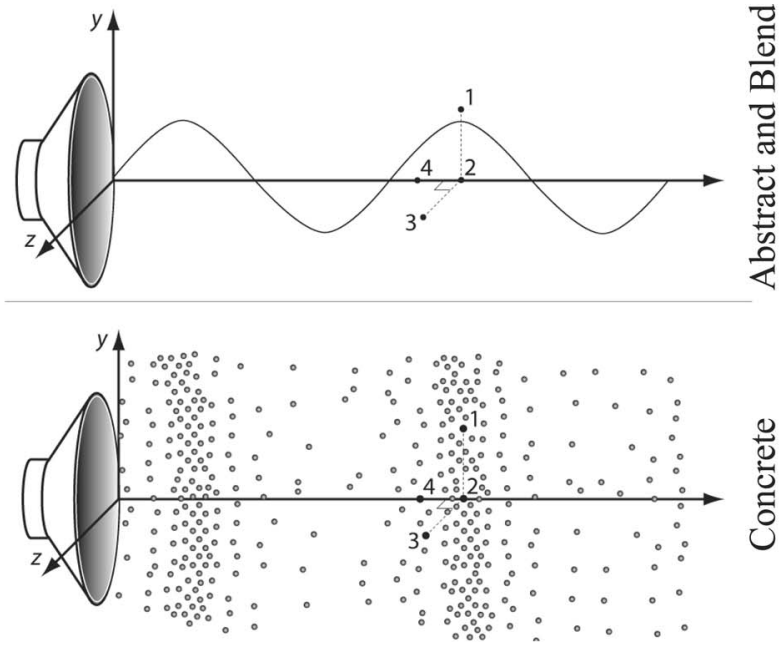

FIG. 9. Question 2 sound waves quiz. The same representation (top) was used for the abstract and blend groups. A different representation (bottom) was used for the concrete group. 


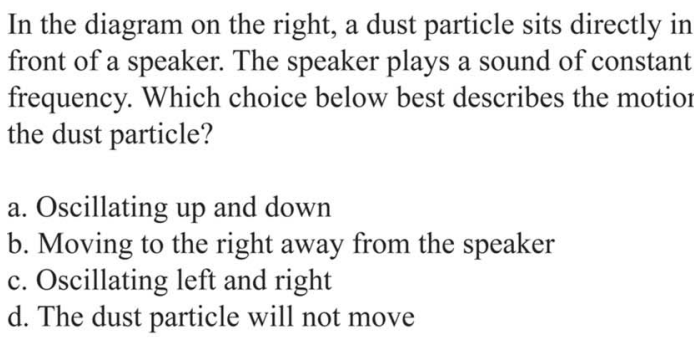

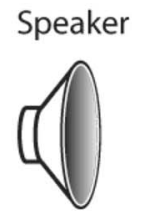

\section{Dust Particle}

o

FIG. 10. Question 3 from the sound waves quiz, identical for all three experimental groups.

blend group. On question 2, WYSIWYG predicts that the concrete group will be likely to answer correctly, since this information can be read directly from the diagram, but does not distinguish between the abstract and blend groups, both of which had the same representation on question 2. WYSIWYG predicts that all three groups will answer similarly (or with similar distributions of answers) on question 3, which was identical in all three groups.

Applying the analogical scaffolding model to question 1, surface-level interpretations of signs couple to associated analogies, and we predict students will respond differently as follows: Students in the abstract group, presented with a sine wave, will be more likely than students in the concrete and blend groups to select analogies that involve vertical motion (e.g., crowd and string analogies). Students in the concrete and blend groups, presented with a picture of air particles, will be more likely than students in the abstract group to select analogies that involve horizontal motion (e.g., slinky and football). ${ }^{53}$ Students' surface-level interpretations of these signs will play key roles for questions 2. Students in the abstract group will be more likely than other groups to use "up means up" reasoning, answering $1>2=4>3$ on question 2. Students in the concrete and blend groups will also use surface-level reasoning, but in this case students will interpret the sign (air particles) as meaning the pressure is the same where the air particle density is the same (and therefore are likely to answer correctly, $1=2=3>4$, or possibly take a more literal reading of the picture and answer $1=2>4=3$ ). Importantly, students in the concrete group can map this information directly from the picture on question 2, while students in the blend group must interpret the sine wave in question 2 as standing for a 3D sound wave. On question 3, absent an overt sign, students' choice of analogy on question 1 will play a key role. Students in the abstract group will more likely answer vertical motion ("up and down") while students in the concrete and blend groups will more likely answer horizontal motion ("to the right" or "left and right").

\section{Results \\ Question 1}

Figure 11 shows the six most popular single (or combination of) analogies selected by students according to their assigned treatment group, accounting for more than $92 \%$ of student responses. Overall, the slinky analogy was the most popular choice, accounting for $43 \%$ of all student answers. ${ }^{54}$ If we include students who selected the slinky analogy in combination with others, we find $66 \%$ of students selected the slinky analogy. We find significant differences, however, between treatment groups in Fig. 11. Students in the concrete and blend groups were significantly more likely to select the slinky analogy than students in the abstract group ( $p$ $<0.01)$. The concrete group was significantly more likely than the abstract group to select both football and slinky in combination $(p=0.03)$, and the abstract group was significantly more likely to select the crowd and string analogies than the other two groups $(p<0.05)$. Thus we observe the predicted association between the representation presented to students and students' choices of analogy.

\section{Question 2}

Figure 12 shows student answers to question 2 according to treatment group, with substantial differences between the three groups. Here, we show the four main answers, accounting for $86 \%$ of student responses. The abstract group was less likely to choose the correct answer $(1=2=3>4)$ than both the blend $(p=0.002)$ and concrete $(p=0.024)$ groups, with the blend group most likely of the three groups to choose the correct answer. Turning to the distracters, stu-

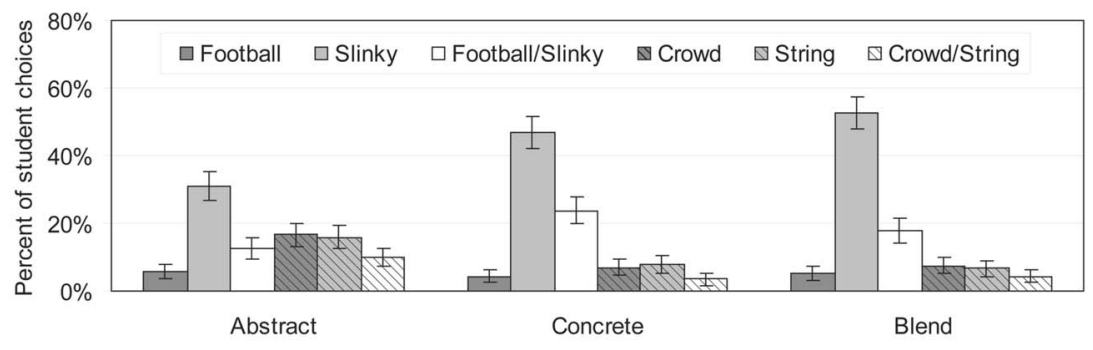

FIG. 11. Percent of students in the abstract, concrete, and blend groups choosing single analogies (football, slinky, crowd, string) or two analogies (football/slinky, crowd/string). Other combinations accounted for less than $8 \%$ of student responses. 


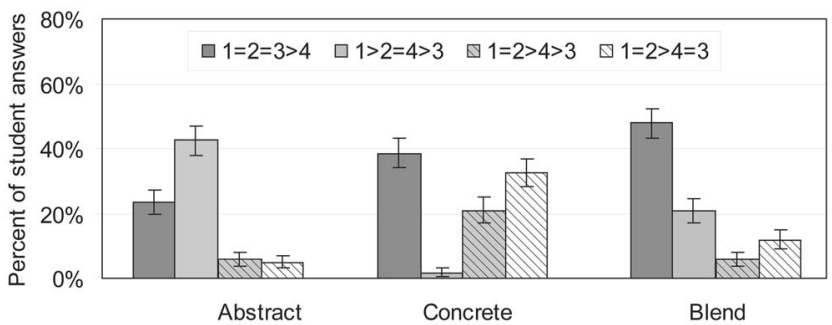

FIG. 12. Student answers to the sound waves quiz question 2 according to experimental group. The correct answer is the leftmost dark gray bar $(1=2=3>4)$.

dents in the abstract group were most likely to select $1>2$ $=4>3(p<0.002)$, followed second by students in the blend group $(p<0.002)$, with students in the concrete group least likely to select this distracter. Students in the concrete group were most likely to select two other distracters, $1=2>4$ $>3(p<0.002)$ or $1=2>4=3(p<0.002)$.

\section{Question 3}

Figure 13 shows student answers to question 3 according to experimental group. Here, we present the three main answers, accounting for $93 \%$ of student responses. The concrete group was marginally more likely to chose the correct answer ("left/right") than the abstract $(p=0.07)$ and blend $(p=0.1)$ groups. On the distracters, the abstract group was more likely than the concrete $(p=0.005)$ and blend $(p$ $=0.064$ ) groups to select "up/down." While we see differences, the effects of changes in representation on student answers to question 3 are limited (generally $p>0.05$ ).

Given the marked effects of representation on question 2, it is noteworthy that we found student responses to question 3 depending only weakly on representation. Notably, the representations presented to students on the first two questions are absent on question 3. To gain some insight, we look within each treatment group to examine how students' analogies (as they selected in question 1) affected their reasoning. Table IV shows the number of students $(N)$ selecting a given analogy (single or multiple) on question 1 above the corresponding analogy, and the fraction of student answers to question 3 below. These associations between analogy and answer are all statistically significant $\left(\chi^{2}, p<0.001\right)$. In the concrete and blend groups, the majority of students selected slinky and/or football analogies, and more than half of these

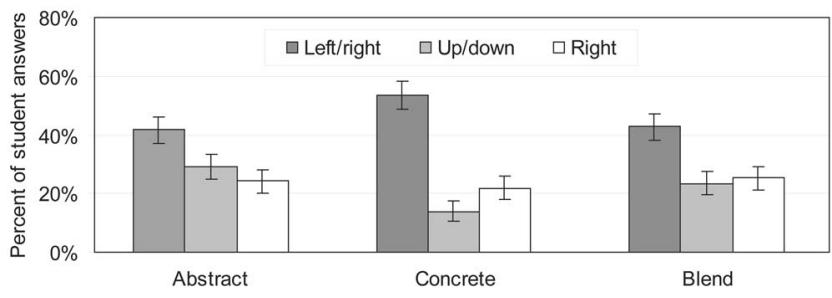

FIG. 13. Student answers to the sound waves quiz question 3 according to experimental group. The correct answer is the leftmost dark gray bar (left/right).

students answered question 3 correctly. Conversely, students in the abstract group tended to select string and/or crowd analogies to a greater degree than student in the other groups. We found that among these students in the abstract group who selected string and/or crowd analogies, 51\% answered "up/down" on question 3. However, a substantial number of students in the abstract group did select slinky and/or football analogies, and, within this select group, 63\% answered question 3 correctly. Interestingly, we found that among the few students who selected only the football analogy, $55 \%$ of these students answered "to the right" on question 3-far more than for any other analogy, and the only group choosing "right" to a considerable degree.

\section{Discussion of the within-domain study}

The purpose of the within-domain study was to examine student reasoning within a single layer, sound waves. According to the analogical scaffolding model, signs can cue productive schemata for students to apply across multiple layers, and in the across-domain study one of these layers involved sound waves. The within-domain study demonstrated three key findings: (i) Signs can drive students' choice of analogy (question 1). (ii) Students in the blend group productively applied the 3D idea to an abstract (sine wave) representation of sound (question 2), while students in the other treatments did not. (iii) Absent an overt sign (question 3), there is only weak association between students' answers and the representations presented on earlier questions. However, we do find a stronger association between students' answers and the analogies they bear in mind.

Thus representation can drive analogy and therefore schemata. We could represent this as an arrow pointing from sign to schema in Fig. 4 and 5, indicating the direction of

TABLE IV. Student answers to question 3 from the sound quiz, split by treatment and analogies selected.

\begin{tabular}{lcccccc}
\hline \hline & \multicolumn{2}{c}{ Abstract } & \multicolumn{2}{c}{ Concrete } & \multicolumn{2}{c}{ Blend } \\
$\mathrm{N}=$ & 59 & 51 & 85 & 21 & 89 & 22 \\
Analogy: & $\begin{array}{c}\text { Slinky and/or } \\
\text { Football }\end{array}$ & $\begin{array}{c}\text { String and/or } \\
\text { Crowd }\end{array}$ & $\begin{array}{c}\text { Slinky and/or } \\
\text { Football }\end{array}$ & $\begin{array}{c}\text { String and/or } \\
\text { Crowd }\end{array}$ & $\begin{array}{c}\text { Slinky and/or } \\
\text { Football }\end{array}$ & $\begin{array}{c}\text { String and/or } \\
\text { Crowd }\end{array}$ \\
\hline Up/down & $10 \%$ & 51 & $11 \%$ & 29 & $15 \%$ & 50 \\
Right & 25 & 22 & 21 & 29 & 27 & 23 \\
Left/right & 63 & 24 & 59 & 38 & 49 & 18 \\
\hline \hline
\end{tabular}


cueing. ${ }^{55}$ (Note that the slinky was the most popular choice in all three treatment groups, and therefore representation is one of several mechanisms driving analogy.) Within the sound waves layer, schemata preferentially cued by abstract and concrete signs were consistent with the predictions of the analogical scaffolding model. For instance, students presented with only an abstract sine wave on the quiz select answers to question 2 reflecting "up means up" reasoning about this representation of a sound wave (i.e., these students tended to select $1>2=4>3$ in Fig. 12). Conversely, students presented with a concrete picture of air particles selected answers reflecting 3D conceptions of a sound wave. However, note that in the concrete treatment, sound was represented by a concrete picture of air particles on question 2 . We might therefore argue that students in the concrete group were able to map this information directly from the diagram shown in Fig. 9. Only students in the blend group interpreted a sine wave as representing a 3D sound wave. According to the analogical scaffolding model, for the blend group, this sine wave took this 3D meaning by way of a prior blend (in question 1) with a concrete picture of air particles. This result confirms the models prediction that schemata that are tightly coupled to concrete signs preferentially project to blends over schemata (weakly) coupled to abstract signs. ${ }^{5}$ If concrete signs were not privileged in this way, we would expect many more students in the blend group to answer similarly to students in the abstract group (on both questions 2 and 3).

Without explicit signs, students' mechanistic reasoning about sound (i.e., motion of air particles) remains strongly coupled to the analogies they bear in mind, as evidenced by Table IV. Interestingly, though students in the abstract group were more likely than other students to use "up means up" reasoning on question 3 (answering "up and down"), the three groups were not significantly different on the correct answer. We may describe this as "weak cueing," whereby the schemata coupled to questions 1 and 2 of the quiz were not strongly cued by the representation (or lack thereof) on question 3. In this situation, students may rely on the analogies they bear in mind (Table IV), or on other prior knowledge of sound. In summary, we note that one indicator of difficulty for students on questions 2 and 3 may be the use of representations. We find students in the abstract group relatively unprepared to interpret abstract representations on question 2 , while students in the blend group demonstrated the highest level of ability to productively interpret these abstract representations.

\section{CONCLUSION}

As part of ongoing studies of student learning with analogy, we have conducted two sets of empirical studies to examine the utility of the analogical scaffolding model. In the first of these studies, we found that analogical scaffolding constitutes a productive tool for analyzing student learning with analogies. In this across-domain study, students taught about EM waves with a tutorial incorporating blends (appro- priately presented according to the analogical scaffolding model) produced response patterns markedly different from students taught the same material without blends. Though our aim in these studies was to observe differential response patterns independent of overall student performance, one cannot escape the conclusion that, of the three treatments examined, the blend treatment is generally more productive of correct student reasoning. Students taught with blends achieved post-test scores three times those of students taught with canonical (abstract) representations alone. In addition, the model explains why tutorials that did not use blends were less beneficial for student learning. Abstract signs (e.g., sine wave) do not always couple to productive schemata, while concrete signs (e.g., air particles) that are coupled to productive schemata do not readily cue these schemata across layers. However, abstract signs do cue productive schemata across layers when blended with a concrete sign in previous layers. Notably, students in the concrete group may be making their own blends.

In a second complementary study, we examined student reasoning about sound waves, demonstrating how blends occur in particular instances. We find that signs can cue particular schemata and associated analogies that appear to drive student reasoning about sound waves. Consistent with the across-domain study, we find abstract signs can couple to unproductive schemata when used alone, but these abstract signs can cue productive schemata when blended previously with a concrete sign. Again, performance differences stand out. On a quiz focusing on sound waves, students presented with blends outperformed (by a factor of 2) students presented with abstract representations alone in their ability to productively interpret these canonical representations.

These across- and within-domain studies provide consistent evidence in support of the weak hypothesis that signs can cue associated, but pre-existing, schemata. This cueing leads to significant variations in student reasoning about waves as measured by the assessments in both studies. Further, the across-domain study provides evidence in support of the strong hypothesis that signs and blending can lead to the formation of new schemata. The various ways these new schemata are formed may depend strongly on the signs used to teach. The across- and within-domain studies support the model of analogical scaffolding and provide a prototype for future studies of this kind.

\section{ACKNOWLEDGMENTS}

This work has been supported by the National Science Foundation Grants No. (NSF DUE-0410744 and NSF DRL0448176), the AAPT/AIP/APS (Colorado PhysTEC program), and the University of Colorado. We wish to extend sincere thanks to Jamie Nagle, Kevin Stenson, and Dale Prull for supporting this work, Edward Redish, Thomas Bing, Michelle Zandieh, Michael Wittmann, and the PER at Colorado Group, particularly Michael Dubson and Patrick Kohl, for essential and significant contributions to this work. We also thank the students for their participation. 
${ }^{1}$ J. Lemke, in Reading Science: Critical and Functional Perspectives on Discourses of Science, edited by J. R. Martin and R. Veels (Routledge, London, 1998).

${ }^{2}$ E. Ochs, P. Gonzales, and S. Jacoby, in Interaction and Grammar, edited by E. Ochs, E. Schegloff, and S. Thompson (Cambridge University Press, Cambridge, England, 1996).

${ }^{3}$ K. Dunbar, in Model-based Reasoning in Scientific Discovery, edited by L. Magnani, N. Nercessian, and P. Thagard (Plenum Press, New York, 1999).

${ }^{4}$ D. Givry and W. M. Roth, Toward a new conception of conceptions: Interplay of talk, gestures, and structures in the setting. J. Res. Sci. Teach. 43, 1086 (2006).

${ }^{5}$ N. S. Podolefsky and N. D. Finkelstein, Analogical scaffolding and the learning of abstract ideas in physics: An example from electromagnetic waves. Phys. Rev. ST Phys. Educ. Res. 3, 010109 (2007).

${ }^{6} \mathrm{M}$. Hesse, Models and Analogies in Science (University of Notre Dame Press, Notre Dame, IN 1966).

${ }^{7} \mathrm{E}$. Rutherford, The scattering of $\alpha$ and $\beta$ particles by matter and the structure of the atom. Philos. Mag. 6, 21 (1911).

${ }^{8}$ Maxwell actually used multiple analogies in addition to fluid theory. See Nancy J. Nersessian, in Reading Natural Philosophy: Essays in the History and Philosophy of Science and Mathematics, edited by David B. Malament (Open Court Publishing Company, Chicago, IL, 2002), pp. 129-166.

${ }^{9}$ M. K. Iding, How analogies foster learning from science texts. Instr. Sci. 25, 233 (1997).

${ }^{10}$ D. Gentner, Structure-mapping: A theoretical framework for analogy. Cogn. Sci. 7, 155 (1983).

${ }^{11}$ K. J. Holyoak and P. Thagard, The analogical mind. Am. Psychol. 52(1), 35 (1997).

${ }^{12}$ D. Gentner and D. R. Gentner, in Mental Models, edited by D. Gentner and A. Stevens (Lawrence Erlbaum Press, Hillsdale, NJ, 1983).

${ }^{13} \mathrm{~S}$. Vosniadou, in Similarity and Analogical Reasoning, edited by S. Vosniadou and A. Ortony (Cambridge University Press, Cambridge, England 1989).

${ }^{14}$ D. E. Brown and J. Clement, Overcoming misconceptions via analogical reasoning: Abstract transfer versus explanatory model construction. Instr. Sci. 18, 237 (1989).

${ }^{15}$ D. Hammer, A. Elby, R. F. Scherr, and E. F. Redish, in Transfer of Learning from a Modern Multidisciplinary Perspective, edited by J. Mestre (Information Age Publishing, Greenwich, CT, 2005), pp. 89-120.

${ }^{16}$ A. A. diSessa, in Constructivism in the Computer Age, edited by G. Forman and P. B. Pufall (Lawerence Erlbaum Associates, Hillsdale, NJ, 1988).

${ }^{17}$ C. Sandifer, Proceedings of the 2003 Physics Education Research Conference, 2004, Vol. 720, pp. 93-96.

${ }^{18} \mathrm{~J}$. Clement, Observed methods for generating analogies in scientific problem solving. Cogn. Sci. 12, 563 (1988).

${ }^{19}$ R. J. Spiro, P. J. Feltovich, R. L. Coulson, and D. K. Anderson, in Similarity and Analogical Reasoning, edited by S. Vosniadou and A. Ortony (Cambridge University Press, Cambridge, England, 1989).

${ }^{20}$ S. M. Glynn, in The Psychology of Learning Science, edited by S. Glynn, R. Yeany and B. Beritton (Erlbaum, Hillsdale, NJ, 1991).

${ }^{21}$ J. Clement, Proceedings of the Fourth Annual Conference of the Cognitive Science Society, 1982, Vol. 4, pp. 79-81.

${ }^{22}$ M. L. Gick and K. J. Holyoak, Analogical problem solving.
Cogn. Psychol. 12, 306 (1980).

${ }^{23}$ M. L. Gick and K. J. Holyoak, Schema induction and analogical transfer. Cogn. Psychol. 15, 1 (1983).

${ }^{24}$ V. M. Sloutsky, J. A. Kaminsky, and A. F. Heckler, The advantage of simple symbols for learning and transfer. Psychonomic Bulletin and Review 12 (3), 508 (2005).

${ }^{25}$ R. L. Goldstone and Y. Sakamoto, The transfer of abstract principles governing complex adaptive systems. Cogn. Sci. 46, 414 (2003).

${ }^{26}$ A. Van Heuvelen and X. Zou, Multiple representations of workenergy processes. Am. J. Phys. 69, 184 (2001).

${ }^{27}$ N. S. Podolefsky and N. D. Finkelstein, Use of analogy in learning physics: The role of representations. Phys. Rev. ST Phys. Educ. Res. 2, 020101 (2006).

${ }^{28}$ B. S. Ambrose, P. R. L. Heron, S. Vokos, and L. C. McDermott, Student understanding of light as an electromagnetic wave: Relating the formalism to physical phenomena. Am. J. Phys. 67, 891 (1999).

${ }^{29}$ Developing well honed curricular materials would require an iterative cycle of design, testing with students, and modification. Analogical scaffolding serves as a cognitive model on which to base curricular materials and may be considered a guide in this iterative development process.

${ }^{30}$ W. M. Roth and G. M. Bowen, Complexities of graphical representations during lectures: A phenomenological approach. Learn. Instr. 9, 235 (1999).

${ }^{31}$ G. Fauconnier and M. Turner, The Way We Think: Conceptual Blending and the Mind's Hidden Complexities (Basic Books, New York, 2003).

${ }^{32}$ G. Lakoff and R. Nunez, Where Mathematics Comes From: How the Embodied Mind Brings Mathematics into Being (Basic Books, New York, 2001).

${ }^{33}$ A. Elby, What students' learning of representation tells us about constructivism. J. Math. Behav. 19, 481 (2000).

${ }^{34} \mathrm{~J}$. Touger, Introductory Physics: Building Understanding (John Wiley \& Sons, New York, 2005).

${ }^{35}$ www.lon-capa.org

${ }^{36}$ Unless otherwise stated, all statistics are based on a two-tailed $z$ test.

${ }^{37}$ L. C. McDermott and P. S. Schaffer, Tutorials in Introductory Physics (Prentice-Hall, Englewood Cliffs, NJ, 2001).

${ }^{38}$ These interviews were conducted as part of the modifications of the materials used in these studies. The standard EM-wave representations often used in textbooks includes crossed $\mathrm{E}$ and $\mathrm{B}$ fields represented by superimposed vectors and sine waves. This standard representation is problematic for several reasons which were revealed in student interviews. In these interviews, we found that students often did not distinguish between the electric and magnetic fields, resulting in false positives on question 2. This is because with the B field shown, students might answer $\mathrm{P}=\mathrm{R}$ since both of these points lie near a wave peak, (the $\mathrm{E}$ field for $\mathrm{P}$ and the $\mathrm{B}$ field for $\mathrm{R})$. We also removed the vectors from these representations in Fig. 2 in order to examine how students make sense of this "stripped down" and more abstract representation.

${ }^{39}$ The idea that, for a traveling wave, a point where the wave crosses the $x$ axis at one instant in time will be nonzero as the wave propagates was covered explicitly for a wave on a string. We expected that, combined with the hint on question 2 in Fig. 2 , students would be able to reason productively about the "time 
averaged" signal of an EM wave. Our classroom observations indicate that students make sense of traveling waves in the context of a wave on a string, but do not, in general, apply this idea when answering question 2 in Fig. 2. We have some evidence that addressing the term "time averaged signal" explicitly helps students make this connection.

${ }^{40}$ Supplementary materials are available at http://per.colorado.edu/ analogy/index.htm

${ }^{41}$ We note that since a surface-level interpretation of a sine wave results in productive ideas for a wave-on-a-string, but not for sound or EM waves, a sine wave can be considered an abstract representation of a sound or EM wave, but relatively concrete for a wave-on-a-string. We purposefully designed this particular "abstract" sound wave representation in order to complement the EM wave representation, (e.g., Fig. 2) and promote student's use of the sound wave analogy in a manner that will facilitate understanding of this EM representation. Additionally, what is labeled as abstract or concrete will depend upon the individual using these representations. See the discussion of abstraction in Ref. 5 and notes (Refs. 48,49) therein.

${ }^{42}$ It may be noted that the PER community recognizes the need for a more refined approach to student thinking than a conceptions or misconceptions approach. However, researchers still argue for the existence of large-scale, stable, consistently activated sets of resources (Ref. 56). For an in depth examination of conceptions, see Elby (Ref. 33).

${ }^{43}$ K. A. Strike and G. J. Posner, in Cognitive Structure and Conceptual Change, edited by L. H. T. West and A. L. Pines (Academic Press, New York, 1985), pp. 211-231.

${ }^{44}$ M. McCloskey, Intuitive physics. Sci. Am. 248, No. 4, p. 122 (1983).

${ }^{45}$ The notion that misconceptions are relatively stable across contexts is testable. For more see Ref. 4.

${ }^{46}$ Along the lines of diSessa's $p$-prims (Ref. 16).

${ }^{47}$ This might be an example of WYSIWYG type reasoning (Ref. 33).

${ }^{48}$ Note that nearly all of the off-diagonal elements, 35 of 36 cells, are zero, (not including the category Other). In this case a $\chi^{2}$ test is invalid. However, because of the nearly perfect diagonalization, we may conclude a strong association between answer choice and stated reasoning.

${ }^{49} \mathrm{We}$ do not have a compelling explanation for the unexpectedly large number of students in the abstract group answering partially correct on the pre-test. Since the majority of these students answered differently, and incorrectly, on the post-test, we consider this result curious, but insignificant to our broader findings.

${ }^{50} \mathrm{We}$ observe $25 \%$ differences between concrete and blend groups on both questions 1 , (correct answer) and 2, (partially correct answer) with $p<0.1$. In this case, since we expect high performers to be less susceptible to representational effects than low performers, a one-tailed $z$ test may be appropriate which would result in $p<0.05$. Note that dividing the student populations into high and low performers reduces $\mathrm{N}$ by half in statistical tests, resulting in reduced significance levels even on observed differences of $25 \%$ between treatments.

${ }^{51}$ In this lab activity, sound was consistently represented by a sine wave, with one pictorial representation of air particles along the lines of the concrete representation used. No blended representations were used.

${ }^{52}$ Z. Hrepic, D. Zollman, and S. Robello, Proceedings of the NARST 2005 Annual Meeting, 2005.

${ }^{53}$ According to the model (Ref. 5), the concrete, (air particles) sign is privileged over the abstract, (sine wave) sign for making meaning of sound. Thus the sine wave inherits the 3D schema from the air particles picture, (and not the other way around).

${ }^{54}$ Note that both transverse and longitudinal waves can be generated on a stretched slinky. Most of the students who choose the slinky analogy indicated in their open response that their choice was associated with a longitudinal wave.

${ }^{55}$ Here, we observe signs driving schemata. Note that schemata may also drive the meaning, (or creation) of signs. We might consider this latter directionality an indicator of expert reasoning.

${ }^{56}$ E. F. Redish, Teaching Physics with the Physics Suite (Wiley, New York, 2003). 